\title{
Maturação da marcha em crianças com paralisia cerebral: Um estudo piloto
}

\author{
Gait maturation in children with cerebral palsy: A pilot study
}

\author{
J.S. Marques, P. Roquetti Fernandes, J. Fernandes Filho, F. B. Policarpo
}

RESUMO

Este estudo se propôs a estudar a maturação da marcha de crianças com paralisia cerebral (PC) bem como a correlação entre os parâmetros maturacionais desta habilidade motora. Para tal, foi aplicado o Podograma e realizada filmagem da marcha em 8 crianças com PC com idade até 7 anos. Foi aplicada a análise de Cluster, dividindo a amostra em dois grupos, sendo a variável relação entre a largura da pelve e afastamento dos tornozelos (REL) utilizada como parâmetro para divisão dos grupos. Utilizouse teste $\mathrm{t}$ de Student, análise dos Deltas e correlação de Pearson. Os resultados demonstraram desvios da normalidade para todos os parâmetros, apresentando maior comprometimento para a velocidade e a cadência, sendo observada correlação significativa entre estes parâmetros, apontando ainda correlação destes parâmetros com o parâmetro REL. Portanto, embora as crianças com paralisia cerebral avaliadas neste estudo tenham adquirido o padrão de deambulação, ainda o realizam de forma imatura.

Palavras-chave: paralisia cerebral, marcha, desenvolvimento infantil

ABSTRACT

This study aimed at observing children with cerebral palsy's (CP) gait maturation and to correlate gait maturation's motor skill parameters. Podogram and video shooting of eight children's gait were used. Children up to seven years of age participated in the study. Cluster analysis was applied, dividing the sample into two groups, using the relation between pelvis' width and the spreading of the ankles (REL) as parameters. Data were analyzed using t-test, analysis of Deltas, and Pearson's correlation. Deviance from normality for all the parameters was demonstrated with greater failure in speed and cadence and a significant correlation among these parameters, and yet between them and the REL parameter. Although children with CP had acquired gait, they still do it immaturely.

Keywords: cerebral palsy, gait, child development

Submetido: 17.05.2010 | Aceite: 31.07.2010

Janice Souza Marques. Universidade Potiguar, Natal - RN; Universidade Castelo Branco, Rio de Janeiro - RJ, Brasil.

Paula Roquetti Fernandes. Centro de Excelência de Avaliação Física, Rio de Janeiro - RJ, Brasil.

José Fernandes Filho. Centro de Excelência de Avaliação Física, Rio de Janeiro - RJ; Universidade Federal do Rio de Janeiro, Rio de Janeiro - RJ, Brasil.

Fernando Policarpo Barbosa. Universidade de Trás-os-Montes e Alto Douro, Vila Real, Portugal.

Endereço para correspondência: Janice Souza Marques - Rua Santa Efigênia, 3317, Candelária, CEP: 59065-590

Natal/RN - Brasil.

E-mail: janicemarques72@gmail.com 
A paralisia cerebral (PC) é uma enfermidade que ocorre em consequência à lesão do sistema nervoso central (SNC) em desenvolvimento, podendo esta lesão ocorrer antes, durante ou após o nascimento (Pato, Souza, \& Leite, 2002). Devido a esta lesão, as crianças portadoras de PC tendem a apresentar diversas alterações tônicas, posturais, de equilíbrio, bem como dificuldade na aquisição dos padrões normais do desenvolvimento humano, dentre eles a marcha. No entanto, mesmo apresentando padrões anormais, o portador de PC pode vir a adquirir a capacidade de deambulação, mesmo que esta se apresente com desvios da normalidade (Bobath \& Bobath, 1989).

A marcha humana surge como consequência ao desenvolvimento do SNC, e depois de adquirida, essa habilidade motora irá passar por um processo de aprimoramento, tornando-se mais madura (Keen, 1993). Sutherland, Olshen, Cooper e Woo (1980) realizaram um estudo com crianças normais com idades até 7 anos que determinou 5 parâmetros indicativos, relacionados à maturação da marcha: percentual de apoio simples, velocidade, cadência, comprimento do passo e relação entre a largura da pelve e afastamento dos tornozelos (REL). Os valores normativos deste estudo permitem analisar a maturação da marcha em diversas enfermidades.

À análise de crianças portadoras de síndrome de Down, Marques, Souza, Araújo e Nogueira (2007) encontraram uma maturação da marcha abaixo dos níveis esperados para a idade cronológica das crianças avaliadas.

Em estudo com portadores de hemiplegia espástica por acidente vascular encefálico, Neves e Nóbrega (2007) também observaram déficits nos 5 parâmetros de maturação da marcha.

O estudo destes parâmetros permite aprofundar o conhecimento dos mecanismos relativos ao desenvolvimento da marcha humana, assim como a compreensão dos estágios maturacionais.
A compreensão e o interesse em se analisar a marcha é bastante antigo e sua importância vem sendo cada vez mais reforçada por ser um recurso que favorece o melhor entendimento do quadro clinico dos pacientes que por ventura tenham alteração na deambulação, auxiliando, assim, nas tomadas de decisão terapêutica (Cook, Schneider, Hazlewood, Hillman, \& Roob, 2003; Davids, 2006; Vásquez, 2004).

Diante do exposto, surgiu o interesse em estudar a marcha de crianças com paralisia cerebral, visando identificar seu estado maturacional, assim como a existência de correlação entre os parâmetros determinantes desta maturação.

\section{MÉTODO}

Este estudo, de caráter descritivo comparativo e correlacional, foi submetido e analisado pelo Comitê de Ética em Pesquisa da Universidade Potiguar (UnP), sendo aprovado (protocolo n. ${ }^{\circ}$ 116/2007) por estar em conformidade com a resolução 196/96 do Conselho Nacional de Saúde.

\section{Amostra}

Foram selecionadas 8 crianças, de ambos os sexos, portadoras de PC, que apresentavam marcha com ou sem apoio e não possuíam enfermidades associadas. Todas as crianças se encontravam em atendimento em uma das seguintes instituições: Clínica Escola de Fisioterapia da Universidade Potiguar (UnP), Centro de Reabilitação Infantil (CRI) e Associação de Pais e Amigos (APAE-RN), todas localizadas na cidade de Natal, Rio Grande do Norte. Solicitou-se aos responsáveis a autorização para a participação das crianças no estudo por meio da assinatura do termo de consentimento livre e esclarecido.

\section{Procedimentos e Instrumentos}

Após autorização, foi feita entrevista com os responsáveis para coleta dos dados clínicos, e na sequência as crianças foram submetidas aos seguintes procedimentos. Antes de realizar a 
marcha, a criança era colocada em decúbito dorsal sob uma maca, quando o avaliador palpou as espinhas ilíacas antero-superiores, e com trena da marca Sanny - Brasil mensurou a distancia entre elas, determinando assim a largura da pelve.

Após a mensuração foi realizada a filmagem da marcha, utilizando 2 máquinas digitais da marcha Sony ${ }^{\circledR}$, uma para filmagem na visão antero-posterior e outra para a captação da marcha em uma visão lateral, estando a criança descalça e com vestimenta que permitisse boa visualização dos membros inferiores. A criança era orientada a deambular livremente sobre uma pista de papel madeira de 4 metros, sendo cronometrado (cronômetro da marca Kenko) o tempo gasto até completar o percurso sobre a pista, para que fosse posteriormente determinada a velocidade utilizando a seguinte equação, seguindo protocolo proposto por Wong et al. (1993):

$$
\begin{gathered}
\mathrm{V}=\mathrm{S} / \mathrm{T} \\
\text { (onde } \mathrm{S}=\text { espaço percorrido e } \mathrm{T}=\text { tempo gasto para } \\
\text { percorrer tal distância) }
\end{gathered}
$$

$\mathrm{Na}$ sequência, o avaliador realizou a contagem de passos realizados durante um minuto, determinando assim a cadência.

Após a filmagem, foi utilizado o Podograma, seguindo protocolo proposto por Jerônimo, Silveira, Borges, Dini e David (2007) e Adams, Chandler e Schuhmann (2000), que consiste na pintura da sola dos pés com tinta atóxica, sendo em seguida solicitada a deambulação em um espaço pré-determinado, delimitado pela pista de papel madeira de 4 metros. As impressões plantares foram depois utilizadas para determinação do comprimento dos passos e afastamento dos tornozelos, conforme protocolo descrito por Rose e Gamble (2007). Utilizando uma trena foi mensurada a distância entre as impressões de dois calcanhares seguidos (do direito ao esquerdo, de forma linear, através de traçado na base dos calcanhares) determinando a variável comprimento do passo. A mensuração da distância entre a impressão dos tornozelos determinava a largura do passo; este escore era então relacionado com o valor obtido com a mensuração da largura da pelve (feita no início da avaliação) para determinação final da variável "relação entre a largura da pelve e a distância entre os tornozelos durante a marcha"(REL).

As filmagens foram posteriormente analisadas para determinar o percentual de apoio simples, que reflete o intervalo onde o indivíduo permanece em apoio unipodal durante o ciclo da marcha. Visando diminuir erro de medida, foram feitas três mensurações, utilizando-se para análise estatística, o valor médio obtido.

\section{Análise Estatística}

Os dados foram tratados estatisticamente utilizando pacote estatístico SPSS, versão 16.0 for Windows. Para verificar normalidade da amostra foi utilizado o teste KolmogorovSmirnov. Seguido da análise de Cluster e do Delta percentual. A comparação das medias foi feita pelo teste $t$ de Student para amostras independentes e posteriormente a análise da correlação de Pearson. Os dados serão apresentados através das medidas de tendência central e dispersão: média e desvio padrão $(M$ $\pm D P)$. Para que se possa estabelecer significância entre os parâmetros analisados, foi observado um valor de $p<.05$.

\section{RESULTADOS}

Os valores absolutos para os parâmetros indicativos de maturação da marcha apresentaram distribuição normal para um $p=$ .05. Os valores médios observados para cada indivíduo podem ser visualizados no quadro 1 .

Através da análise de Clusters, a amostra foi dividida em grupo $1(\mathrm{G} 1 ; n=5)$ e grupo 2 $(\mathrm{G} 2 ; n=3)$. Para tal divisão foi utilizado como parâmetro a variável "relação entre a largura de pelve e afastamento dos tornozelos" (REL), devido a mesma ser a única variável de maturação que não apresentava escores para comparação individual. 
Quadro 1

Valores obtidos para os parâmetros de marcha por indivíduo com paralisia cerebral

\begin{tabular}{ccccccc}
\hline Indivíduo & $\begin{array}{c}\text { Idade } \\
(\text { anos })\end{array}$ & $\begin{array}{c}\text { APS } \\
(\%)\end{array}$ & $\begin{array}{c}\text { VEL } \\
(\mathrm{cm} / \mathrm{s})\end{array}$ & $\begin{array}{c}\text { CAD } \\
(\mathrm{pas} / \mathrm{min})\end{array}$ & $\begin{array}{c}\text { PASSO } \\
(\mathrm{cm})\end{array}$ & $\begin{array}{c}\text { REL } \\
(\mathrm{cm})\end{array}$ \\
\hline 1 & 3 & 59.5 & 136.1 & 101.6 & 69.5 & 1.6 \\
2 & 3 & 54.4 & 13.3 & 32.8 & 31.0 & 6.4 \\
3 & 4 & 68.0 & 79.4 & 67.7 & 53.0 & 1.6 \\
4 & 2 & 70.2 & 86.2 & 83.9 & 46.0 & 1.9 \\
5 & 7 & 66.0 & 50.8 & 40.8 & 58.0 & 9.0 \\
6 & 6 & 75.7 & 115.2 & 56.9 & 49.0 & 1.4 \\
7 & 4 & 60.6 & 61.1 & 66.1 & 36.0 & 2.5 \\
8 & 6 & 53.2 & 116.2 & 74.0 & 92.0 & 2.0 \\
\hline
\end{tabular}

Nota: APS: percentual de apoio simples; VEL: velocidade; CAD: cadência; PASSO: comprimento do passo; REL: relação entre a largura da pelve e a distância entre os tornozelos durante a marcha.

A média de idades do G1 foi de $4.20 \pm 1.78$ anos (Mín $=2$ e Máx $=6$ ), enquanto que no G2 a média foi de $4.67 \pm 2.08$ anos (Mín $=3$ e Máx $=7$ ).

Os valores médios observados para G1 foram maiores em relação ao G2 em quase todos os parâmetros de maturação da marcha. No entanto, o valor médio obtido para a razão da largura da pelve com o afastamento dos tornozelos para os dois grupos não apresentou diferença significativa $(p>.05)$. Dentre os cinco parâmetros considerados para o estudo da marcha, quando comparados observou-se que apenas a velocidade apresentou diferença significativa entre os grupos (ver quadro 2 ).

Quadro 2

Distribuição e comparação dos parâmetros de maturação de marcha das crianças com paralisia cerebral analisadas

\begin{tabular}{cccc}
\hline & $\begin{array}{c}\text { Grupo 1 } \\
(n=5)\end{array}$ & $\begin{array}{c}\text { Grupo 2 } \\
(n=3)\end{array}$ & $p$ \\
& $M \pm D P$ & $M \pm D P$ & \\
\hline Idade & $4.20 \pm 1.78$ & $4.67 \pm 2.08$ & .74 \\
APS & $65.33 \pm 8.91$ & $60.32 \pm 5.77$ & .42 \\
VEL & $106.59 \pm 23.39$ & $41.72 \pm 25.16$ & .01 \\
CAD & $76.79 \pm 16.98$ & $46.54 \pm 7.37$ & .05 \\
PASSO & $61.90 \pm 19.11$ & $41.66 \pm 14.36$ & .16 \\
REL & $1.68 \pm .25$ & $1.68 \pm 7.72$ & 1.0 \\
\hline
\end{tabular}

Nota: APS: percentual de apoio simples; VEL: velocidade; CAD: cadência; PASSO: comprimento do passo; REL: relação entre a largura da pelve e a distância entre os tornozelos durante a marcha.

Quando relacionados os parâmetros, foi observada correlação significativa entre a velocidade e a cadência $(r=.80)$. Não obstante, o parâmetro REL foi correlacionado significativamente com as variáveis, velocidade e cadência $(r=-.72$ e $r=-.75$, respectivamente) (ver quadro 3 ).

Quadro 3

Correlação entre os parâmetros de maturação de marcha das crianças com paralisia cerebral analisadas

\begin{tabular}{cccccc}
\hline & REL & APS & VEL & CAD & PASSO \\
\hline REL & 1.0 & -.206 & $-.721^{*}$ & $-.746^{*}$ & -.226 \\
APS & - & 1.0 & .209 & .026 & -.281 \\
VEL & - & - & 1.0 & $.802^{*}$ & .689 \\
CAD & - & - & - & 1.0 & .468 \\
PASSO & - & - & - & - & 1.0 \\
\hline
\end{tabular}

$* p<.05$

Nota: REL: relação entre a largura da pelve e a distância entre os tornozelos durante a marcha; APS: percentual de apoio simples; VEL: velocidade; CAD: cadência; PASSO: comprimento do passo.

A análise do delta percentual determinou os desvios da normalidade (DN) dos parâmetros de maturação da marcha (ver quadro 4). Foram observados maiores desvios com relação ao 
padrão de cadência e menores desvios para o padrão percentual de apoio simples, indicando que as crianças do G1 apresentam uma maturação da marcha correspondente a crianças de 2 anos, e que as do G2 estão no estágio de maturação da marcha de uma criança de 3 anos.

Quadro4

Desvios da normalidade dos parâmetros de marcha por criança com paralisia cerebral (deltas percentuais)

\begin{tabular}{crrrr}
\hline Indiv. & $\begin{array}{c}\text { DN do } \\
\text { APS }\end{array}$ & $\begin{array}{c}\text { DN da } \\
\text { VEL }\end{array}$ & $\begin{array}{c}\text { DN da } \\
\text { CAD }\end{array}$ & $\begin{array}{c}\text { DN do } \\
\text { PASSO }\end{array}$ \\
\hline 1 & -6.0 & 50.1 & -84.5 & -65.2 \\
2 & -11.1 & -72.7 & -123.0 & -73.2 \\
3 & 4.3 & -20.6 & -99.0 & -76.3 \\
4 & 3.4 & 14.2 & -110.0 & -53.0 \\
5 & 3.6 & -63.2 & -85.0 & -87.5 \\
6 & 12.1 & 6.2 & -97.0 & -87.9 \\
7 & -3.1 & -38.9 & -116.0 & -75.4 \\
8 & -10.4 & 7.2 & -54.0 & -87.3 \\
\hline
\end{tabular}

Nota: DN: desvio da normalidade; APS: percentual de apoio simples; VEL: velocidade; CAD: cadência; PASSO: comprimento do passo.

\section{DISCUSSÃO}

Este estudo teve como objetivo analisar a maturação da marcha de crianças com paralisia cerebral, buscando ainda encontrar a correlação entre os parâmetros determinantes desta maturação. Foi encontrado que, mesmo tento adquirido o padrão de marcha, as crianças com PC o desempenham com desvios da normalidade, refletindo imaturidade deste padrão motor. Percebeu-se ainda que as crianças analisadas demonstraram maiores comprometimentos para a velocidade e a cadência, parâmetros estes que apresentaram correlação entre si e de ambos com o parâmetro REL.

Os resultados de desvios da normalidade para os parâmetros de maturação, encontrados nas 8 crianças portadoras de paralisia cerebral analisadas, convergem com outros estudos que indicam valores maiores para os parâmetros espaço-temporais da marcha em portadores de paralisia cerebral (Abel \& Damiano, 1996; Holt, Fonseca, \& LaFiandra, 2000; Jerônimo et al., 2007). Embora não tenha ocorrido diferença estatisticamente significativa para a maioria dos parâmetros, o fato das maiores médias terem ocorrido no G1 pode ser explicado devido a este grupo ser mais jovem, estando assim em níveis maturacionais abaixo dos indivíduos do G2.

A maturação da marcha ocorre à medida que vai ocorrendo a maturação do sistema nervoso central, refletindo um aprimoramento do padrão motor de deambulação. Para crianças normais, acredita-se que por volta dos 7 anos os aspectos relativos à aquisição da marcha já apresentem semelhança com os do adulto (Vásquez, 2005). Já em indivíduos que possuam alguma enfermidades que afete $o$ processo de maturação neurológica serão observados desvios no desenvolvimento (Caram, Funayama, Spina, Giuliani, \& Neto, 2006; Largo, Molinari, Weber, Comenale, \& Duc, 1985), porém isto não implicará na impossibilidade de adquirir habilidades necessárias para a deambulação, sendo observado que os portadores de enfermidades conseguem desenvolver a locomoção, embora isso ocorra de forma mais lenta que nos indivíduos normais (Mancini et al., 2002). Estudo realizado por Ferreira (2008) com portadores de PC do tipo hemiparético encontrou valores abaixo do esperado para os parâmetros de maturação de marcha, assim como os obtidos neste estudo.

Os valores médios semelhantes entre os grupos para o padrão relação da largura da pelve com o afastamento dos tornozelos podem ter ocorrido em consequência desta relação se apresentar aproximadamente constante quando a criança chega aos 3 anos (Sutherland, 1997). Embora os valores esperados para normalidade sejam maiores que os apresentados pelos indivíduos analisados, tal fato demonstra que, mesmo com suas limitações e adaptações, o individuo portador de PC irá apresentar a sequência neuroevolutiva esperada para o desenvolvimento normal. A redução nos valores absolutos deste padrão pode ser explicada pelo padrão de 
adução observado comumente nestes indivíduos, o que gera uma marcha com pouco afastamento dos tornozelos. Tal fato corrobora com os escritos de Sanz (2003) sobre a presença de marcha patológica mediante padrões de espasticidade.

A diferença significativa para o padrão de velocidade ao se comparar os grupos pode ser explicada pela presença de indivíduos com menor comprometimento motor no G1. Crianças portadoras de PC leve evoluem mais rapidamente que aquelas que apresentam níveis moderados e severos (Cury et al., 2006; Tieman, Palisano, Gracely, \& Rosenbaum, 2007; Wu, Day, Strauss, \& Shavelle, 2004). Desta forma, adquirem mais precocemente a marcha e por consequência sua maturação ocorre mais previamente. Riad, Akerlind e Miller (2007) encontraram valores maiores para a velocidade das crianças portadoras de PC ao comparar seus resultados com outras crianças portadoras da mesma enfermidade, mas que possuíam idades menores.

A correlação observada entre os parâmetros velocidade e cadência corrobora com os achados na literatura. À medida que o individuo vai adquirindo maior habilidade na realização da marcha, vai aumentando a velocidade de sua execução e por consequência o numero de passos realizados em determinado período de tempo, ou seja, aumenta a cadência. $O$ estudo realizado por Jerônimo et al. (2007) descreve tal relação como estratégia de aprimoramento da marcha quando utilizada estimulação elétrica funcional em portadores de PC do tipo hemiparético. No entanto, estudo utilizando toxina botulínica A encontrou diminuição da cadência mediante aumento da velocidade (Faria, Masiero, Spósito, \& Saad, 2001).

A correlação negativa entre o parâmetro REL e as variáveis velocidade e cadência é também indicativa do aprimoramento da marcha pois, ao se aumentar a velocidade e cadência, o indivíduo tende a apresentar diminuição da base de sustentação (Desloovere et al., 2006), o que reflete um amadurecimento da marcha, reforçando o que foi discutido anteriormente e estando de acordo com relatos de estudos relacionados ao tema, que afirmam haver a possibilidade de evolução do portador de PC mesmo mediante alterações motoras (Bell, Õunpuu, DeLuca, \& Romness, 2002).

O menor desvio de normalidade de $6.3 \%$ em média observado para o padrão de percentual de apoio simples indica que este parâmetro é o menos afetado no processo de maturação da marcha na amostra estudada. Tal situação pode ser explicada pelo fato das crianças analisadas não apresentarem distúrbios de equilíbrio tão severos, estando assim de acordo com Zatsiorsky, Werner e Kaimim (1994) que afirmam que, quanto menor o distúrbio de equilíbrio, mais próximo do normal o percentual de apoio simples apresentado pelo indivíduo.

Foram observados maiores desvios para velocidade e cadência $(34.14 \%$ e $96.60 \%$, em média, respectivamente), indicando que os indivíduos analisados apresentam marcha mais lenta e realizam poucos passos por minuto. A inexistência de tabela normativa para o parâmetro REL, não permitiu determinar o delta para este parâmetro, o que pode considerado como outro ponto limitante do estudo, pois impossibilitou a determinação do desvio para este, que é um dos cinco parâmetros de maturação da marcha.

Os desvios da normalidade presentes em todos os parâmetros apresentados pelos indivíduos demonstram que mesmo tendo adquirido o padrão de deambulação, as crianças portadoras de paralisia cerebral a realizam com desvios da normalidade, tentando assim compensar suas limitações e se tornarem funcionais.

Os parâmetros de maturação da marcha mais afetados no portador de PC foram a velocidade e a cadência, no entanto os indivíduos analisados tentam compensar esta dificuldade mesmo mediante bases de sustentação diminuídas. 


\section{CONCLUSÕES}

Os resultados obtidos no presente estudo apontam para a seguinte conclusão: os indivíduos portadores de PC estudados apresentaram comprometimento da marcha em todos os parâmetros quando comparados com tabela normativa, indicando imaturidade deste padrão motor; no entanto apenas o parâmetro velocidade mostrou diferença significativa ao comparar os grupos, bem como apresentou correlação com a cadência e ainda com o parâmetro relação entre a largura da pelve e afastamento dos tornozelos. Embora haja alteração destes parâmetros, observou-se que a capacidade de deambulação foi adquirida independente da lesão, indicando assim o próprio processo de maturação biológico inerente ao desenvolvimento da marcha.

Acredita-se que o entendimento de como se apresenta a maturação da marcha das crianças com paralisia cerebral possa favorecer $o$ direcionamento do plano terapêutico que vise promoção de funcionabilidade e independência para estas crianças por parte de diversos profissionais que atuam com esta população como fisioterapeutas, educadores físicos, ortopedistas, entre outros, que devem atuar de forma conjunta, unindo os dados obtidos com seus conhecimentos específicos tentando todos atingir o objetivo comum de reabilitação do indivíduo. Para o futuro, espera-se que a análise dos parâmetros de maturação da marcha seja utilizada tanto para acompanhamento quanto para decisão de alta do paciente, tornando-se um procedimento realizado de forma rotineira nas práticas clínicas. Sugere-se reprodução do estudo em amostras maiores bem como realizar uma comparação dos parâmetros de maturação com os níveis de comprometimento motor apresentado pelos indivíduos.

\section{REFERÊNCIAS}

Abel, M. F., \& Damiano, D. L. (1996). Strategies for increasing walking speed in diplegic cerebral palsy. Journal of Pediatric Orthopaedics, 16(6), 753-758.
Adams, M. A., Chandler, L. S., \& Schuhmann K. (2000). Gait changes in children with cerebral palsy following a neurodevelopmental treatment course. Pediatric Physical Therapy, 12 (3), 114-120.

Bell, K. J., Õunpuu, S., DeLuca, P. A., \& Romness, M. J. (2002). Natural progression of gait in children with cerebral palsy. Journal of Pediatric Orthopaedics, 22, 677-682.

Bobath, B., \& Bobath, K. (1989). Desenvolvimento motor nos diferentes tipos de paralisia cerebral. São Paulo: Manole.

Caram, L. H. A., Funayama, C. A. R., Spina, C. I., Giuliani, L. R., \& Neto, J. M. P. (2006). Investigação das causas de atraso no neurodesenvolvimento: Recursos e desafios. Arquivos de Neuropsiquiatria, 64(2B), 466-472.

Cook, R. E., Schneider, I., Hazlewood, M. E., Hillman, S. J., \& Roob, J. E. (2003). Gait analysis alters decision-making in cerebral palsy. Journal of Pediatric Orthopaedics, 23, 292295.

Cury, V. C. R., Mancini, M. C., Melo, A. P., Fonseca, S. T., Sampaio, R. F., \& Tirado, M. G. A. (2006). Efeitos do uso de órtese na mobilidade funcional de crianças com paralisia cerebral. Revista Brasileira de Fisioterapia, 10(1), 67-74.

Davids, J. R. (2006). Quantitative gait analysis in the treatment of children with cerebral palsy. Journal of Pediatric Orthopaedics, 26 (4), 557-559.

Desloovere, K., Molenaers, G., Feys, H., Huenaerts, C., Callewaert, B., \& Walle, P. V. (2006). Do dynamic and static clinical measurements correlate with gait analysis parameters in children with cerebral palsy? Gait \& Posture, 24(3), 302-313.

Faria, T. C. C., Masiero, D., Spósito, M. M. M., \& Saad, M. (2001). A avaliação do uso da toxina botulínica A e da cinesioterapia na melhora da marcha do portador de paralisia cerebral do tipo hemiparético. Acta Fisiátrica, 8(3), 101-106.

Ferreira, M. G. (2008). Análise da maturação da marcha em portadores de paralisia cerebral do tipo hemiplégico/hemiparético: Estudo de casos. Trabalho de Conclusão de Curso de Graduação em Fisioterapia, Universidade Potiguar, Natal, Brasil.

Holt, K. G., Fonseca, S. T., \& LaFiandra, M. E. (2000). The dynamics of gait in children with spastic hemiplegic cerebral palsy: Theoretical and clinical implication. Human Movement Science, 19(3), 375-405. 
Jerônimo, B. P., Silveira, J. A., Borges, M. B. S., Dini, P. D., \& David, A. C. (2007). Variáveis espaçotemporais da marcha de crianças com paralisia cerebral submetidas a eletroestimulação no músculo tibial anterior. Revista Brasileira de Fisioterapia, 11(4), 261-266.

Keen, M. (1993). Early development and attainment of normal mature gait. Journal of Prosthetics $\mathcal{E}$ Orthotics, 5(2), 35-38.

Largo, R. H., Molinari, L., Weber, M., Comenale, P. L., \& Duc, G. (1985). Early development of locomotion: Significance of prematurity, cerebral palsy and sex. Developmental Medicine $\mathcal{E}$ Child Neurology, 27, 183-191.

Mancini, M. C., Fiúza, P. M., Rebelo, J. M., Magalhães, L. C., Coelho, Z. A. C., Paixão, M. L., ... Fonseca, S. T. (2002). Comparação do desempenho de atividades funcionais em crianças com desenvolvimento normal e crianças com paralisia cerebral. Arquivos de Neuropsiquiatria, 60(2B), 446-452.

Marques, J. S., Souza, E. L. B., Araújo, A. S., \& Nogueira, J. I. C. (2007). Evaluation of marching in down syndrome children carriers. The FIEP Bulletin, 77, 157-160.

Neves, F. C., \& Nóbrega, M. L. T. (2007). Análise da maturação da marcha em pacientes acometidos de acidente vascular encefálico isquêmico em fase crônica. Trabalho de Conclusão de Curso de Graduação em Fisioterapia, Universidade Potiguar, Natal, Brasil.

Pato, T. R., Souza, D. R., \& Leite, H. P. (2002). Epidemiologia da paralisia cerebral. Acta Fisiátrica, 9(2), 71-76.

Riad, J., Akerlind, Y. H., \& Miller, F. (2007). Classification of spastic hemiplegic cerebral palsy in children. Jornal of Pediatric Orthopaedics, 27(7), 758-764.

Rose, J., \& Gamble, J. G. (2007). Marcha: Teoria e prática da locomoção humana ( $3^{\mathrm{a}}$ ed.). Rio de Janeiro: Guanabara Koogan.

Sanz, C. M. (2003). Marcha patológica. Revista del Pie y Tobillo, 17(1), 1-7.

Sutherland, D. H. (1997). The development of mature gait. Gait \& Posture, 6(2), 163-170.

Sutherland, D. H., Olshen, P. L., Cooper. L., \& Woo, S. L. (1980). The development of mature gait. The Journal of Bone E Joint Surgery, 62A(3), 336353.

Tieman, B., Palisano, R. J., Gracely, E. J., \& Rosenbaum, P. L. (2007). Variability in mobility of children with cerebral palsy. Pediatric Physical Therapy, 19, 180-187.

Vásquez, S. C. (2004). La marcha: Historia de los procedimentos de análisis. Revista de la Facultad de Ciências de la Salud, 2, 1-15.

Vásquez, S. C. (2005). Desarrollo de la marcha. Revista de la Facultad de Ciências de la Salud, 3, 115.

Wong, A. M. K., Chen, C. L., Hong, W. G., Chion, W. K., Chen, H. C., \& Tang, F. T. (1999). Gait analysis through foot patterns recognition for children with cerebral palsy. Journal of Musculoskeletal Research, 3(1), 71-81.

Wu, Y. W., Day, S. M., Strauss, D. J., \& Shavelle, R. M. (2004). Prognosis for ambulation in cerebral palsy: A population-based study. Pediatrics, 114(5), 1264-1271.

Zatsiorsky, V. M., Werner, S. L., \& Kaimim, M. A. (1994). Basic kinematics of walking: Step length and step frequency - A review. The Journal of Sports Medicine and Physical Fitness, 34(2), 109-134.

(c) EY-No Todo o conteúdo da revista Motricidade está licenciado sob a Creative Commons, excepto quando especificado em contrário e nos conteúdos retirados de outras fontes bibliográficas. 